\title{
Pacific
}

Journal of

Mathematics

\section{ON THE FROBENIUS MORPHISM OF FLAG SCHEMES}

MASAHARU KANEDA 


\title{
ON THE FROBENIUS MORPHISM OF FLAG SCHEMES
}

\author{
MAsaharu Kaneda \\ Dedicated to Professor C. W. Curtis on the occasion of his 65th birthday
}

\begin{abstract}
We give a new proof to V. B. Mehta and A. Ramanthan's theorem that the Schubert subschemes in a flag scheme are all simultaneously compatibly split, using the representation theory of infinitesimal algebraic groups. In particular, the present proof dispenses with the Bott-Samelson schemes.
\end{abstract}

Let $K$ be a perfect field of positive characteristic $p$. If $A$ is a $K$-algebra and $r \in \mathbf{Z}$, one defines a new $K$-algebra $A^{(r)}$ by the ring homomorphism $K \rightarrow A$ such that $\xi \mapsto \xi^{p^{-r}}$. Given a $K$-scheme $\mathfrak{X}$ we will denote by $\mathfrak{X}^{(r)}$ the $K$-scheme having the same underlying topological space as that of $\mathfrak{X}$ but with the structure sheaf $\mathscr{O}_{\mathfrak{X}} \otimes_{K} K^{(-r)}$, which we regard as a sheaf of $K$-algebras by the usual multiplication of $K$ on $K^{(-r)}$ from the right. If $\mathscr{F}$ is an $\mathscr{O}_{\mathfrak{X}}$-module, we set $\mathscr{F}(r)=\mathscr{F} \otimes_{K} K^{(-r)}$; it comes equipped with the structure of an $\mathscr{O}_{\mathfrak{X}^{(r)}}$ module. If $r>0$, the morphism $F_{\mathfrak{X}}^{r}: X \rightarrow X^{(r)}$ that is the identity on the underlying topological spaces and such that $a \otimes \xi \mapsto a^{p^{r}} \xi$ for each $a \in \Gamma\left(\mathfrak{V}, \mathscr{O}_{\mathfrak{X}}\right)$ and $\xi \in K^{(-r)}$ with $\mathfrak{V}$ open in $\mathfrak{X}$ is called the $r$ th Frobenius morphism of $\mathfrak{X}$.

If $K$ is algebraically closed, Hartshorne [HASV], (III.6.4) showed that on the projective spaces over $K$, the direct image of any invertible sheaf under the Frobenius morphism splits into a direct sum of invertible sheaves; this was crucial for B. Haastert [Haas] to prove the $\mathscr{D}$-affinity of the projective spaces. We will compute in $\S 1$ which invertible sheaf enters as a direct summand.

More generally, we say after V. B. Mehta and A. Ramanathan [MR] that $\mathfrak{X}$ is Frobenius split iff the structural morphism $F_{\mathfrak{X}}^{\mathfrak{f}}: \mathscr{O}_{\mathfrak{X}^{(1)}} \rightarrow$ $F_{\mathfrak{X} *} \mathscr{O}_{\mathfrak{X}}$ admits a left inverse, called a Frobenius splitting, so that $\mathscr{O}_{\mathfrak{X}^{(1)}}$ is a direct summand of $F_{\mathfrak{X} *} \mathscr{O}_{\mathfrak{X}}$. If $\sigma$ is a Frobenius splitting of $\mathfrak{X}$ and if $\mathfrak{Y}$ is a closed subscheme of $\mathfrak{X}$ defined by an ideal sheaf $\mathscr{I}$, we say $\sigma$ splits $\mathfrak{Y}$ iff $\sigma\left(F_{\mathfrak{X} *} \mathscr{I}\right) \subseteq \mathscr{I}^{(1)}$, in which case $\mathfrak{Y}$ will also be Frobenius split, said to be compatibly split in $\mathfrak{X}$.

Mehta and Ramanathan showed that the flag schemes are Frobenius split with all the Schubert subschemes compatibly split. Their 
result has various applications, e.g., to their simple proof of Kempf's (resp. Demazure's) vanishing theorem of the higher cohomology of dominant (resp. ample) invertible sheaves on the flag schemes (resp. the Schubert schemes).

In $\S 3$ we will rederive a part of their theorem that the flag schemes are Frobenius split, using the representation theory of infinitesimal algebraic groups. Along the same line one can find a particularly nice splitting of each flag scheme that splits all its Schubert subschemes; that we will do in $\S 4$.

We will let $K$ Alg $\left(\right.$ resp. Mod $_{\mathfrak{X}}$ ) denote the category of $K$-algebras (resp. $\mathscr{O}_{\mathfrak{X}}$-modules). Also $\mathbf{S c h}_{K}$ (resp. $\mathbf{G r p}_{K}$ ) is the category of $K$ schemes (resp. $K$-group schemes). If $\mathfrak{G}$ is a $K$-group, $\mathfrak{G} \operatorname{Mod}$ will denote the category of $\mathfrak{G}$-modules.

The $\S 4$ is largely due to the referee, who kindly communicated a sketch of the arguments. We have also revised the proof in (3.2) of the surjectivity of a nonzero $G_{r} B$-homomorphism from $S t_{r} \otimes_{K} S t_{r}$ into $\widehat{Z}_{r}\left(2\left(p^{r}-1\right) \rho\right)$. Formerly the argument was borrowed from Jantzen's book [J], (II.11.13).

The author is grateful to the referee for generously sharing his/her ideas with him. Thanks are also due to Akiyama S. for a helpful suggestion to (1.3).

1. Projective spaces. In this section we assume $K$ is algebraically closed and consider the case $\mathfrak{X}=\mathbf{P}^{N}$ the projective $N$-space over $K$.

(1.1) As $\mathbf{P}^{N}$ is defined over $\mathbf{F}_{p},\left(\mathbf{P}^{N}\right)^{(1)} \simeq \mathbf{P}^{N}$. We will denote by $F$ the composite of $F_{\mathrm{P}^{N}}$ with the isomorphism.

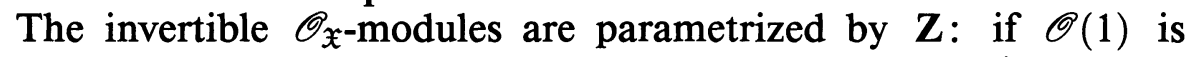
Serre's twisting sheaf, we let $\mathscr{O}(n)=\mathscr{O}(1)^{\otimes_{n}}\left(\right.$ resp. $\left.\mathscr{O}(-n)^{-1}\right)$ if $n \geq 0$ (resp. $n<0)$.

By [HASV], (III.6.4) for any $n \in \mathbf{Z}$ there are $n_{i} \in \mathbf{Z}$ such that

$$
F_{*} \mathscr{O}(n) \simeq \coprod_{i=0}^{p^{N}-1} \mathscr{O}\left(n_{i}\right) \text { in } \operatorname{Mod}_{\mathfrak{X}}
$$

We will compute the $n_{i}$ in this section.

(1.2) If $n=n^{\prime}+p n^{\prime \prime}$ with $n^{\prime} \in[0, p-1]$ and $n^{\prime \prime} \in \mathbf{Z}$, then

$$
F_{*} \mathscr{O}(n) \simeq F_{*}\left(\mathscr{O}\left(n^{\prime}\right) \otimes_{\mathscr{O}_{\circledast}} F^{*} \mathscr{O}\left(n^{\prime \prime}\right)\right) \simeq F_{*} \mathscr{O}\left(n^{\prime}\right) \otimes_{\mathscr{O}_{\mathfrak{X}}} \mathscr{O}\left(n^{\prime \prime}\right)
$$

by the projection formula; hence we have only to compute $F_{*} \mathscr{O}(n)$, 
$n \in[0, p-1]$. Fix such $n$. Then (cf. [Haas], p. 400)

$$
\exists \theta_{i} \in \mathbf{N} \text { with } \sum_{i \geq 0} \theta_{i}=p^{N}: F_{*} \mathscr{O}(n)=\coprod_{i \geq 0}\left(\mathscr{O}(-i)^{\oplus_{\theta_{i}}}\right) .
$$

Let $S_{m}$ be the $m$ th homogeneous part of the polynomial algebra in $N+1$ indeterminates over $K$. Then for each $j \in \mathbf{N}$ we have as $K$-linear spaces

$$
\text { (3) } \begin{aligned}
S_{n+j p} & \simeq \Gamma(\mathfrak{X}, \mathscr{O}(n+j p)) \simeq \operatorname{Mod}_{\mathfrak{X}}\left(\mathscr{O}_{\mathfrak{X}}, \mathscr{O}(n+j p)\right) \\
& \simeq \operatorname{Mod}_{\mathfrak{X}}\left(F^{*} \mathscr{O}_{\mathfrak{X}}, \mathscr{O}(n+j p)\right) \\
& \simeq \operatorname{Mod}_{\mathfrak{X}}\left(\mathscr{O}_{\mathfrak{X}}, F_{*} \mathscr{O}(n+j p)\right) \\
& \simeq \operatorname{Mod}_{\mathfrak{X}}\left(\mathscr{O}_{\mathfrak{X}}, \coprod_{i} \mathscr{O}(j-i)^{\oplus_{\theta_{i}}}\right) \quad \text { by the projection formula } \\
& \simeq \coprod_{i} S_{j-i}^{\oplus_{\theta_{l}}},
\end{aligned}
$$

hence

$$
\left(\begin{array}{c}
n+j p+N \\
N
\end{array}\right)=\sum_{i} \theta_{i}\left(\begin{array}{c}
j-i+N \\
N
\end{array}\right)
$$

In order to compute the $\theta_{i}$, we will agree that for each $t \in \mathbf{Z}$ and $m \in \mathbf{N}$

(5) $\left(\begin{array}{c}t \\ m\end{array}\right)=\left.\frac{1}{m !} \frac{d^{m}}{d x^{m}}\right|_{x=1} x^{t}= \begin{cases}1 & \text { if } m=0 \\ \frac{t(t-1) \cdots(t-m+1)}{m !} & \text { if } m \geq 1\end{cases}$

(1.3) Lemma. (i) For each $r \in \mathbf{N}$

$$
\theta_{r}=\sum_{i=0}^{r}(-1)^{i}\left(\begin{array}{c}
N+1 \\
i
\end{array}\right)\left(\begin{array}{c}
n+(r-i) p+N \\
N
\end{array}\right)
$$

(ii) If $r \geq N+1$ or $n+N \geq(N+1-r) p$, then $\theta_{r}=0$.

(iii) $\theta_{N}= \begin{cases}\left(\begin{array}{c}p-n-1 \\ N\end{array}\right) & \text { if } p-n-1 \geq N, \\ 0 & \text { otherwise. }\end{cases}$

Proof. (i) We will argue by induction on $r$. If $r=0$, take $j=0$ in (1.2) (4) to verify the assertion. If $r \geq 1$, take $j=r$ in (1.2)(4) to 
get

(1)

$$
\begin{aligned}
\theta_{r}= & \left(\begin{array}{c}
n+r p+N \\
N
\end{array}\right)-\sum_{i=0}^{r-1} \theta_{i}\left(\begin{array}{c}
r-i+N \\
N
\end{array}\right) \\
= & \left(\begin{array}{c}
n+r p+N \\
N
\end{array}\right) \\
& -\sum_{s=0}^{r-1}\left(\begin{array}{c}
n+s p+N \\
N
\end{array}\right) \sum_{k=0}^{r-1-s}(-1)^{k}\left(\begin{array}{c}
N+1 \\
k
\end{array}\right)\left(\begin{array}{c}
r-s-k+N \\
N
\end{array}\right)
\end{aligned}
$$

by the induction hypothesis; hence one has only to show

(2) $-\sum_{k=0}^{t-1}(-1)^{k}\left(\begin{array}{c}N+1 \\ k\end{array}\right)\left(\begin{array}{c}t-k+N \\ N\end{array}\right)=(-1)^{t}\left(\begin{array}{c}N+1 \\ t\end{array}\right) \quad \forall t \in[1, r]$.

Assume first $t-1 \leq N$. Then the left-hand side of (2) is

$$
-\sum_{k=0}^{N+1}(-1)^{k}\left(\begin{array}{c}
N+1 \\
k
\end{array}\right)\left(\begin{array}{c}
t-k+N \\
N
\end{array}\right)+(-1)^{t}\left(\begin{array}{c}
N+1 \\
t
\end{array}\right)
$$

hence it will be enough to show

$$
\sum_{k=0}^{N+1}(-1)^{k}\left(\begin{array}{c}
N+1 \\
k
\end{array}\right)\left(\begin{array}{c}
t-k+N \\
N
\end{array}\right)=0 \quad \forall t \geq 1 .
$$

But the left-hand side is

$$
\begin{aligned}
&\left.\sum_{k=0}^{N+1}(-1)^{k}\left(\begin{array}{c}
N+1 \\
k
\end{array}\right) \frac{1}{N !} \frac{d^{N}}{d x^{N}}\right|_{x=1} x^{t-k+N} \\
&=\left.\frac{1}{N !} \frac{d^{N}}{d x^{N}}\right|_{x=1}\left\{x^{t-1}(x-1)^{N+1}\right\}=0,
\end{aligned}
$$

using the Leibniz rule.

If $t-1>N$, then the left-hand side of (2) is

$$
-\sum_{k=0}^{N+1}(-1)^{k}\left(\begin{array}{c}
N+1 \\
k
\end{array}\right)\left(\begin{array}{c}
t-k+N \\
N
\end{array}\right)=0 \text { by (4) }
$$

while the right-hand side of (2) is 0 as $t \geq N+2$. Hence (i) holds.

(ii) If $r \geq N+1$, then

$$
\begin{aligned}
\theta_{r} & =\sum_{i=0}^{N+1}(-1)^{i}\left(\begin{array}{c}
N+1 \\
i
\end{array}\right)\left(\begin{array}{c}
n+(r-i) p+N \\
N
\end{array}\right) \text { by }(\mathrm{i}) \\
& =\left.\frac{1}{N !} \frac{d^{N}}{d x^{N}}\right|_{x=1}\left\{x^{n+(r-N+1) p+N}(x-1)^{p(N+1)}\right\}=0,
\end{aligned}
$$

using the Leibniz rule again. Likewise the rest. 
(1.4) We summarize the foregoing computations in

Proposition. If $n \in[0, p-1]$ and $n^{\prime} \in \mathbf{N}$, then

$$
F_{*} \mathscr{O}\left(n+p n^{\prime}\right) \simeq \coprod_{i=0}^{N} \mathscr{O}\left(n^{\prime}-i\right)^{\oplus_{\theta_{i}}} \quad \text { in } \operatorname{Mod}_{\mathfrak{X}}
$$

with $\theta_{i}=\sum_{j=0}^{i}(-1)^{j}\left(\begin{array}{c}N+1 \\ j\end{array}\right)\left(\begin{array}{c}n+(i-j) p+N \\ N\end{array}\right)$ as in (1.3).

2. Preliminaries. In this section we recall some standard facts of the Frobenius splittings and of the representation theory of algebraic groups. We will also introduce the notations in (2.5) to be used in $\S 3$ and $\S 4$.

(2.1) Let $\mathfrak{X}$ be a $K$-scheme. If $\mathfrak{V}$ is open in $\mathfrak{X}$, one can identify $\mathscr{O}_{\mathfrak{X}^{(1)}}(\mathfrak{V})=\Gamma\left(\mathfrak{V}, \mathscr{O}_{\mathfrak{X}^{(1)}}\right)$ with $\mathscr{O}_{\mathfrak{X}}(\mathfrak{V})^{(1)}$. Then the structure morphism $F_{\mathfrak{X}}^{\mathfrak{f}}(\mathfrak{V}): \mathscr{O}_{\mathfrak{X}^{(1)}}(\mathfrak{V}) \rightarrow\left(F_{\mathfrak{X} *} \mathscr{O}_{\mathfrak{X}}\right)(\mathfrak{V})$ is just the $p^{r}$ th power map. Hence a Frobenius split $K$-scheme is reduced [R], Remark 1.3(i).

(2.2) LemMA (cf. [R], Corollary 1.11 and [MR], Lemma 1). Let $\mathfrak{X}$ be a K-scheme Frobenius split by $\sigma \in \operatorname{Mod}_{\mathfrak{X}^{(1)}}\left(F_{\mathfrak{X} *} \mathscr{O}_{\mathfrak{X}}, \mathscr{O}_{\mathfrak{X}^{(1)}}\right)$.

(i) If $\mathfrak{X}_{1}$ and $\mathfrak{X}_{2}$ are closed subschemes of $\mathfrak{X}$ both split by $\sigma$, then so is $\mathfrak{X}_{1} \cap \mathfrak{X}_{2}$.

(ii) Let $\mathfrak{Y}$ be a closed subscheme of $\mathfrak{X}$ split by $\sigma$. If the underlying space $|\mathfrak{Y}|$ of $\mathfrak{Y}$ is Noetherian, then each irreducible component of $\mathfrak{Y}$ given the reduced closed structure is also split by $\sigma$.

Proof. (i) If $\mathscr{I}_{i}$ is the ideal sheaf of $\mathfrak{X}_{i}$, the ideal sheaf of $\mathfrak{X}_{1} \cap \mathfrak{X}_{2}$ is $\mathscr{I}_{1}+\mathscr{I}_{2}$. Then

$$
\sigma\left(F_{\mathfrak{X} *}\left(\mathscr{J}_{1}+\mathscr{I}_{2}\right)\right) \subseteq \mathscr{I}_{1}^{(1)}+\mathscr{I}_{2}^{(1)}=\left(\mathscr{I}_{1}+\mathscr{I}_{2}\right)^{(1)},
$$

and hence $\mathfrak{X}_{1} \cap \mathfrak{X}_{2}$ is split by $\sigma$.

(ii) If $|\mathfrak{Y}|=\left|\mathfrak{Y}_{1}\right| \cup \cdots \cup\left|\mathfrak{Y}_{r}\right|$ is a decomposition into the irreducible components of $\mathfrak{Y}$, each of which is given the reduced closed structure, put $\mathfrak{V}=|\mathfrak{X}| \backslash\left(\left|\mathfrak{Y}_{2}\right| \cup \cdots \cup\left|\mathfrak{Y}_{r}\right|\right)$. Then $\left|\mathfrak{Y}_{1} \cap \mathfrak{V}\right|=|\mathfrak{Y} \cap \mathfrak{V}|$. As both $\mathfrak{Y}_{1} \cap \mathfrak{V}$ and $\mathfrak{Y} \cap \mathfrak{V}$ are reduced, $\mathfrak{Y}_{1} \cap \mathfrak{V}=\mathfrak{Y} \cap \mathfrak{V} ;$ hence

$$
\mathfrak{Y}_{1} \cap \mathfrak{V} \text { is split by }\left.\sigma\right|_{\mathfrak{V}^{(1)}} \text { in } \mathfrak{V} \text {. }
$$

Let $\mathscr{P}$ be the ideal sheaf of $\mathfrak{Y}_{1}$ in $\mathfrak{X}$. To see that $\sigma\left(F_{\mathfrak{X} *} \mathscr{P}\right) \subseteq \mathscr{P}^{(1)}$, the problem being local we may assume $\mathfrak{X}=\mathfrak{S p}_{K} A$ for some $K$ algebra $A$. Then $\mathscr{P}=\tilde{\mathfrak{p}}$ and $\sigma\left(F_{\mathfrak{X} *} \mathscr{P}\right)=\widetilde{\mathfrak{I}}$ for some ideals $\mathfrak{p}$ and 
$\mathfrak{I}$ of $A$ with $\mathfrak{p} \subseteq \mathfrak{I}$. As $\mathfrak{Y}_{1}$ is reduced and irreducible, $\mathfrak{p}$ is prime. By (2) there is $f \in A \backslash \mathfrak{p}$ such that $\mathfrak{p}_{f}=\mathfrak{I}_{f}$ in $A_{f}$; hence $\mathfrak{I}=\mathfrak{p}$, as desired.

(2.3) Let $\mathfrak{G}$ be an affine algebraic $K$-group scheme, $\mathfrak{H}$ a subgroup scheme of $\mathfrak{G}$, and $\pi: \mathfrak{G} \rightarrow \mathfrak{G} / \mathfrak{H}$ the quotient morphism. $\mathfrak{G} / \mathfrak{H}$ is a $K$-scheme (cf. [J], $(\mathbf{I} .5 .6)(8)$ ), and $\pi$ is open and affine (cf. [J], $(\mathrm{I} .5 .7)(3),(1))$.

If $M$ is an $\mathfrak{H}$-module and if $\mathfrak{V}$ is open in $\mathfrak{G} / \mathfrak{H}$, we set

$$
\begin{aligned}
\operatorname{Sch}_{K}\left(\pi^{-1} \mathfrak{V}, M\right)^{\mathfrak{H}} & \\
=\left\{f \in \mathbf{S c h}_{K}\left(\pi^{-1} \mathfrak{V}, M\right) \mid\right. & \\
& f(A)(x h)=h^{-1} f(A)(x) \\
& \forall x \in\left(\pi^{-1} \mathfrak{V}\right)(A) \\
& h \in \mathfrak{H}(A), A \in K \mathbf{A l g}\} .
\end{aligned}
$$

One defines an $\mathscr{O}_{\mathfrak{G} / \mathfrak{H}}$-module $\mathscr{L}_{\mathfrak{G} / \mathfrak{H}}(M)$ by

$$
\mathfrak{V} \mapsto \mathbf{S c h}_{K}\left(\pi^{-1} \mathfrak{V}, M\right)^{\mathfrak{H}}
$$

The correspondence $M \mapsto \mathscr{L}_{\mathfrak{G} / \mathfrak{H}}(M)$ defines an exact functor from $\mathfrak{H}$ Mod into the category of quasicoherent $\mathscr{O}_{\mathfrak{G} / \mathfrak{h}}$-modules. $\mathscr{L}_{\mathfrak{G} / \mathfrak{H}}(M)$ carries also a structure of $\mathfrak{G}$-linearization.

If we let $\mathfrak{H}$ operate on the coordinate algebra $K[\mathfrak{G}]$ of $\mathfrak{G}$ (resp. $M$ ) by the right regular action (resp. as given), and take the $\mathfrak{H}$-fixed point set of $M \otimes_{K} K[\mathfrak{G}]$, we get a left exact functor

$$
\text { ind }_{\mathfrak{H}}^{\mathfrak{G}}: \mathfrak{H} \operatorname{Mod} \rightarrow \mathfrak{G} \text { Mod via } M \mapsto\left(M \otimes_{K} K[\mathfrak{G}]\right)^{\mathfrak{H}},
$$

where the $\mathfrak{G}$-module structure on $\left(M \otimes_{K} K[\mathfrak{G}]\right)^{\mathfrak{H}}$ is given by the left regular action on $K[\mathfrak{G}]$. Then

$$
\operatorname{ind}_{\mathfrak{H}}^{\mathfrak{G}}(M) \simeq \Gamma\left(\mathfrak{G} / \mathfrak{H}, \mathscr{L}_{\mathfrak{G} / \mathfrak{H}}(M)\right) \text { in } \mathfrak{G} \text { Mod. }
$$

The functor $\operatorname{ind}_{\mathfrak{H}}^{\mathfrak{G}}$ is right adjoint to the forgetful functor $\mathfrak{G} \operatorname{Mod} \rightarrow$ $\mathfrak{H}$ Mod: If $V$ is a $\mathfrak{G}$-module, one has a $K$-linear isomorphism

$$
\mathfrak{G} \operatorname{Mod}\left(V, \operatorname{ind}_{\mathfrak{H}}^{\mathfrak{G}}(M)\right) \rightarrow \mathfrak{H} \operatorname{Mod}(V, M) \quad \text { via } f \mapsto e_{M} \circ f
$$

with an inverse given by $g \mapsto \hat{g}$ such that

$$
\begin{aligned}
\hat{g}(v)(A)(x)=\left(g \otimes_{K} A\right)\left(x^{-1}(v \otimes 1)\right), & \\
v & \in V, x \in \mathfrak{G}(A), A \in K \text { Alg, }
\end{aligned}
$$

where $e_{M}=M \otimes_{K} \varepsilon_{\mathfrak{G}} \in \mathfrak{H} \operatorname{Mod}\left(\operatorname{ind}_{\mathfrak{H}}^{\mathfrak{G}}(M), M\right)$ such that $\sum m_{i} \otimes a_{i} \mapsto$ $\sum \varepsilon_{\mathfrak{G}}\left(a_{i}\right) m_{i}$ with $\varepsilon_{\mathfrak{G}}$ the counit of the Hopf algebra $K[\mathfrak{G}]$, or $e_{M}$ is the evaluation at the neutral element of $\mathfrak{G}(K)$ under the identification 
(4). The isomorphism (5) is called a Frobenius reciprocity. One has also the tensor identity (cf. [J], (I.3.6)) in $\mathfrak{G}$ Mod :

$$
V \otimes_{K} \operatorname{ind}_{\mathfrak{H}}^{\mathfrak{G}}(M) \stackrel{\sim}{\rightarrow} \operatorname{ind}_{\mathfrak{H}}^{\mathfrak{G}}\left(V \otimes_{K} M\right)
$$

such that the image of $v \otimes f$ sends $x \in \mathfrak{G}(A)$ into $\left(x^{-1}(v \otimes 1)\right) \otimes_{A} f(x)$, $A \in K$ Alg .

(2.4) Let $\mathfrak{K}$ be a subgroup of $\mathfrak{H}$ and $q: \mathfrak{G} / \mathfrak{K} \rightarrow \mathfrak{G} / \mathfrak{H}$ the natural morphism. One has (cf. [J], (I.5.19)(5))

$$
\mathscr{L}_{\mathfrak{G} / \mathfrak{H}} \circ \text { ind }{ }_{\mathfrak{K}}^{\mathfrak{H}} \simeq q_{*} \mathscr{L}_{\mathfrak{G} / \mathfrak{K}} \text { on } \mathfrak{K} \operatorname{Mod}
$$

such that if $\mathfrak{V}$ is an affine open of $\mathfrak{G} / \mathfrak{H}$ and $M \in \mathfrak{K}$ Mod, the following commutative diagram results:

$$
K\left[\pi^{-1} \mathfrak{V}\right] \otimes_{K} \operatorname{ind}_{\mathfrak{K}}^{\mathfrak{H}}(M) \longrightarrow K\left[\pi^{-1} \mathfrak{V}\right] \otimes_{K} M
$$

$$
\operatorname{Sch}_{K}\left(\pi^{-1} \mathfrak{V}, \operatorname{ind}_{\mathfrak{K}}^{\mathfrak{H}}(M)\right)^{\mathfrak{H}} \stackrel{\sim}{\longrightarrow} \operatorname{Sch}_{K}\left(\pi^{-1} \mathfrak{V}, M\right)^{\mathfrak{K}}
$$

where $\pi$ is the quotient morphism $\mathfrak{G} \rightarrow \mathfrak{G} / \mathfrak{H}$ and the top horizontal map is given by $a \otimes f \mapsto a \otimes e_{M}(f)$.

Taking the global sections of (1) yields the transitivity of inductions:

$$
\operatorname{ind}_{\mathfrak{H}}^{\mathfrak{G}} \circ \operatorname{ind}_{\mathfrak{K}}^{\mathfrak{H}} \simeq \operatorname{ind}_{\mathfrak{K}}^{\mathfrak{H}} \text {. }
$$

If $L \in \mathfrak{K}$ Mod, the transitivity of inductions makes the following diagram commute:

$$
\begin{array}{ccc}
\operatorname{ind}_{\mathfrak{H}}^{\mathfrak{G}}\left(\operatorname{ind}_{\mathfrak{K}}^{\mathfrak{H}}(L)\right) \stackrel{\sim}{\longrightarrow} \underset{\operatorname{ind}_{\mathfrak{K}}^{\mathfrak{f}}(L)}{\longrightarrow} & \operatorname{ind}_{\mathfrak{K}}^{\mathfrak{G}}(L) \\
\operatorname{ind}_{\mathfrak{K}}^{\mathfrak{H}}(L) & & \downarrow e_{L} \\
& & L .
\end{array}
$$

(2.5) We now fix the notations to be used throughout the rest of the paper. $G$ will denote a semisimple simply connected $K$-group with a maximal torus $T$, both split over $\mathbf{Z}$, and $R$ the root system of $G$ relative to $T$ with a positive system $R^{+}$. We choose a Borel subgroup $B$ of $G$ containing $T$ such that the roots of the unipotent radical $U$ of $B$ are $-R^{+}$, and set $\mathfrak{X}=G / B$.

Let $W=N_{G}(T) / T$ be the Weyl group of $G$. If $\alpha$ is a simple root, let $s_{\alpha}$ be the reflexion in $W$ associated to $\alpha$, and let $l: W \rightarrow \mathbf{N}$ be the length function on $W$ with respect to $\left\{s_{\alpha} \mid \alpha\right.$ simple $\}$. If $w_{0} \in$ $W$ with $w_{0} R^{+}=-R^{+}$, set $U^{+}=w_{0} U w_{0}^{-1}$. Then $\left\{w U^{+} B\right\}_{w \in W}$ provides an open covering of $G$. 
As $B=T \ltimes U, \operatorname{Grp}_{K}\left(B, G L_{1}\right) \simeq \operatorname{Grp}_{K}\left(T, G L_{1}\right)$, which we will denote by $X$. $X$ has the structure of an abelian group, called the weight lattice, such that $(\lambda+\mu)(t)=\lambda(t) \mu(t), t \in T, \lambda, \mu \in X$. Define a partial order on $X$ such that $\lambda \leq \mu$ iff $\mu-\lambda \in \sum_{\alpha \in R^{+}} \mathbf{N} \alpha$. Let $X^{+}$be the set of dominant weights, and put $\rho=\frac{1}{2} \sum_{\alpha \in R^{+}} \alpha \in X^{+}$.

If $M$ is a $T$-module, one can write $M=\coprod_{\lambda \in X} M_{\lambda}$ with $M_{\lambda}=$ $\{m \in M \mid t(m \otimes 1)=m \otimes \lambda(t) \quad \forall t \in T(A), A \in K \mathbf{A l g}\}$. We say $\lambda$ is a weight of $M$ iff $M_{\lambda} \neq 0$.

By abuse of notation we let $\lambda \in X$ also denote the 1-dimensional $B$-module defined by $\lambda$. One has (cf. [J], (II.2.6))

$$
\operatorname{ind}_{B}^{G}(\lambda) \neq 0 \text { iff } \lambda \in X^{+},
$$

in which case (cf. [J],$($ II. 2.2))

(2) $\operatorname{ind}_{B}^{G}(\lambda)$ has the highest weight $\lambda$ with $\operatorname{dimind}_{B}^{G}(\lambda)_{\lambda}=1$.

If $G_{r}=\operatorname{ker} F_{G}^{r}, F_{\mathfrak{X}}^{r}: \mathfrak{X} \rightarrow \mathfrak{X}^{(r)}$ factors through the natural morphism $q: \mathfrak{X} \rightarrow G / G_{r} B$ to induce an isomorphism $F: G / G_{r} B \rightarrow \mathfrak{X}^{(r)}$ in $\mathbf{S c h}_{K}$ (cf. [J], (I.9.5)) so that the diagram

$$
\begin{aligned}
\mathfrak{X} & \stackrel{F_{\mathfrak{X}}^{r}}{\longrightarrow} \mathfrak{X}^{(r)} \\
q \downarrow & \sim \nearrow F \\
G / G_{r} B &
\end{aligned}
$$

commutes. If $B_{r}=\operatorname{ker} F_{B}^{r}$ and $U_{r}^{+}=\operatorname{ker} F_{U^{+}}^{r}$, the multiplication induces an isomorphism of $K$-schemes $U_{r}^{+} \times B_{r} \rightarrow G_{r}$ (cf. [J], (II.3.2)).

For simplicity we set

$$
\widehat{Z}_{r}=\operatorname{ind}_{B}^{G_{r} B}: B \operatorname{Mod} \rightarrow G_{r} B \text { Mod. }
$$

As $G_{r} B / B \simeq U_{r}^{+}$is affine,

$$
\widehat{Z}_{r} \text { is exact (cf. [J], (I.5.13)). }
$$

If $\lambda \in X$, then (cf. [J], (II.9.2))

$$
\widehat{Z}_{r}(\lambda) \text { has highest weight } \lambda \text { with } \operatorname{dim} \widehat{Z}_{r}(\lambda)_{\lambda}=1
$$

and

$$
\widehat{Z}_{r}(\lambda)^{*} \simeq \widehat{Z}_{r}\left(-\lambda+2\left(p^{r}-1\right) \rho\right) \text { in } G_{r} B \text { Mod. }
$$

Also (cf. [J], (II.9.5))

$$
\operatorname{soc}_{G_{r} B} \widehat{Z}_{r}(\lambda) \text { is simple of highest weight } \lambda \text {. }
$$


In particular (cf. [J], (II.3.18)),

$$
\begin{aligned}
& \widehat{Z}_{r}\left(\left(p^{r}-1\right) \rho\right) \text { is simple and admits a structure } \\
& \text { of } G \text {-module, }
\end{aligned}
$$

called the $r$ th Steinberg module and denoted $S t_{r}$.

One has by $(2.4)(1)$

$$
q_{*} \mathscr{L}_{G / B}(M) \simeq \mathscr{L}_{G / G_{r} B}\left(\widehat{Z}_{r}(M)\right) \quad \forall M \in B \text { Mod. }
$$

As $B$ is defined over $\mathbf{F}_{p}, B^{(r)} \simeq B$ in $\mathbf{G r p}_{K}$ (cf. [J], (I.9.5)); hence one can make $M$ into a $G_{r} B$-module, denoted $M^{[r]}$, through the quotient morphism $G_{r} B \rightarrow G_{r} B / G_{r}$ composed with the isomorphism $G_{r} B / G_{r} \sim B^{(r)}$ induced by $F_{G}^{r}$. One has

$$
F_{*} \mathscr{L}_{G / G_{r} B}\left(M^{[r]}\right) \simeq \mathscr{L}_{G / B}(M)^{(r)} \quad \operatorname{inMod}_{\mathfrak{X}^{(r)}}
$$

That is given in each $\left(w U^{+} B / B\right)^{(r)}, w \in W$, by the following commutative diagram:

$$
\begin{aligned}
& \Gamma\left(\left(w U^{+} B / B\right)^{(r)}, F_{*} \mathscr{L}_{G / G_{r} B}\left(M^{[r]}\right)\right) \stackrel{\sim}{\longleftarrow} \Gamma\left(\left(w U^{+} B / B\right)^{(r)}, \mathscr{L}_{G / B}(M)^{(r)}\right) \\
& \text { l) }
\end{aligned}
$$

$$
\begin{array}{ccc}
\operatorname{Sch}_{K}\left(w U^{+}, M^{[r]}\right)^{U_{r}^{+}} & \operatorname{Sch}_{K}\left(w U^{+}, M\right) \otimes_{K} K^{(-r)} \\
\Downarrow & 4 \\
K\left[w U^{+}\right]_{r}^{U_{r}^{+}} \otimes_{K} M^{[r]} & \longleftarrow M \otimes_{K}\left(K\left[w U^{+}\right] \otimes_{K} K^{(-r)}\right)
\end{array}
$$

with the bottom horizontal map given by $m \otimes a \otimes \xi \mapsto a^{p^{r}} \xi \otimes m$.

(2.6) We examine next the inverse image $q^{*} \mathscr{L}_{G / G_{r} B}(V), \quad V \in$ $G_{r} B$ Mod. As the quotient morphism $G \rightarrow G / G_{r} B$ is not locally trivial, the argument of $[\mathbf{J}],(\mathbf{I} .5 .17)(1)$ does not apply as it is. One could consult [CPS], (3.1.2) and (2.7), but we prefer to write down an explicit proof of the following fact:

Proposition. Let $s \in \mathbf{N}, r \in \mathbf{Z}^{+}$, and let $q_{s}: G / G_{s} B \rightarrow G / G_{r+s} B$ be the natural morphism. If $V$ is a $G_{r+s} B$-module, the imbedding

$$
\mathscr{L}_{G / G_{r+s} B}(V) \rightarrow q_{s *} \mathscr{L}_{G / G_{s} B}(V)
$$

induces an isomorphism

$$
q_{s}^{*} \mathscr{L}_{G / G_{r+s} B}(V) \rightarrow \mathscr{L}_{G / G_{s} B}(V)
$$


that makes, in each $w U^{+} x_{s}=w U^{+} B / G_{s} B, w \in W$, the following diagram commutative:

$$
\Gamma\left(w U^{+} x_{s}, q_{s}^{*} \mathscr{L}_{G / G_{r+s} B}(V)\right) \stackrel{\sim}{\longrightarrow} \Gamma\left(w U^{+} x_{s}, q_{s}^{*} \mathscr{L}_{G / G_{s} B}(V)\right)
$$

$K\left[w U^{+} / U_{s}^{+}\right] \otimes_{K\left[w U^{+} / U_{r+s}^{+}\right]}\left(K\left[w U^{+}\right] \otimes_{K} V\right) \longrightarrow \quad K\left[w U^{+}\right] \otimes_{K} V$

where the bottom horizontal map is given by $b \otimes c \otimes v \mapsto b c \otimes v$.

Proof. By taking the direct limit we may assume $\operatorname{dim} V<\infty$. Let $\pi_{s}: G \rightarrow G / G_{s} B$ and $\pi_{s}^{\prime}: G \rightarrow G / G_{r+s} B$ be the quotient morphisms so that $q_{s} \circ \pi_{s}=\pi_{s}^{\prime}$. Define $\psi: q_{s}^{*} \mathscr{L}_{G / G_{r+s} B}(V) \rightarrow \mathscr{L}_{G / G_{s} B}(V)$ to be the adjoint of the imbedding $\mathscr{L}_{G / G_{r+s} B}(V) \rightarrow q_{s *} \mathscr{L}_{G / G_{s} B}(V)$.

Assume first $s=0$. As $\left\{w U^{+} x_{0}^{r+s}\right\}_{w \in W}$ is an open covering of $\mathfrak{X}$, to see that $\psi$ is invertible, we have only to check it in each $w U^{+} x_{0}$, $w \in W$, then only in $U^{+} x_{0}$ by the $W$-equivariance; hence it is enough to show that the map

$$
\begin{aligned}
\Gamma\left(U^{+} x_{0}, \mathscr{O}_{\mathfrak{X}}\right) \otimes_{\Gamma\left(U^{+} x_{r}, \mathscr{O}_{G / G_{r} B}\right)} \operatorname{Sch}_{K}\left(U^{+} B, V\right)^{G_{r} B} \\
\quad \rightarrow \mathbf{S c h}_{K}\left(U^{+} B, V\right)^{B}
\end{aligned}
$$

is invertible. But the left-hand side is isomorphic to

$$
\begin{aligned}
K\left[U^{+}\right] & \otimes_{K\left[U^{+} / U_{r}^{+}\right]} \operatorname{Sch}_{K}\left(U^{+}, V\right)^{U_{r}^{+}} \\
& \simeq K\left[U^{+}\right] \otimes_{K\left[U^{+}\right]^{(r)}} \text { ind }_{U_{r}^{+}}^{U^{+}}(V) \\
& \simeq K\left[U^{+}\right] \otimes_{K\left[U^{+}\right]^{(r)}}\left(V \otimes_{K} K\left[U^{+}\right]\right)_{r}^{U_{r}^{+}}
\end{aligned}
$$

while the right-hand side is isomorphic to $V \otimes_{K} K\left[U^{+}\right]$. Hence we are reduced to showing that the map

$$
\begin{array}{r}
K\left[U^{+}\right] \otimes_{K\left[U^{+}\right]^{(r)}}\left(V \otimes_{K} K\left[U^{+}\right]\right) \rightarrow V \otimes_{K} K\left[U^{+}\right] \\
\text {via } a \otimes m \otimes b \mapsto m \otimes a b
\end{array}
$$

induces an isomorphism upon restriction to

$$
K\left[U^{+}\right] \otimes_{K\left[U^{+}\right]^{(r)}}\left(V \otimes_{K} K\left[U^{+}\right]\right)^{U_{r}^{+}} .
$$

We will argue by induction on $\operatorname{dim} V$. Note that

$$
V^{U_{r}^{+}} \neq 0 \text { if } V \neq 0
$$

as $U_{r}^{+}$is unipotent. In particular, if $\operatorname{dim} V=1, V=V^{U_{r}^{+}}$; hence

$$
\left(V \otimes_{K} K\left[U^{+}\right]\right)^{U_{r}^{+}} \simeq V \otimes_{K}\left(K\left[U^{+}\right]^{U_{r}^{+}}\right) \simeq V \otimes_{K} K\left[U^{+}\right]^{(r)},
$$

and the assertion follows. 
Assume next $\operatorname{dim} V>1$. As $U^{+} / U_{r}^{+} \simeq U^{(r)}$ is affine, ind $U_{U^{+}}^{U^{+}}$is exact. Also $K\left[U^{+}\right]$is free of rank $p^{r\left|R^{+}\right|}$over $K\left[U^{+}\right]^{(r)}$. Hence we get a commutative diagram of column exact sequences
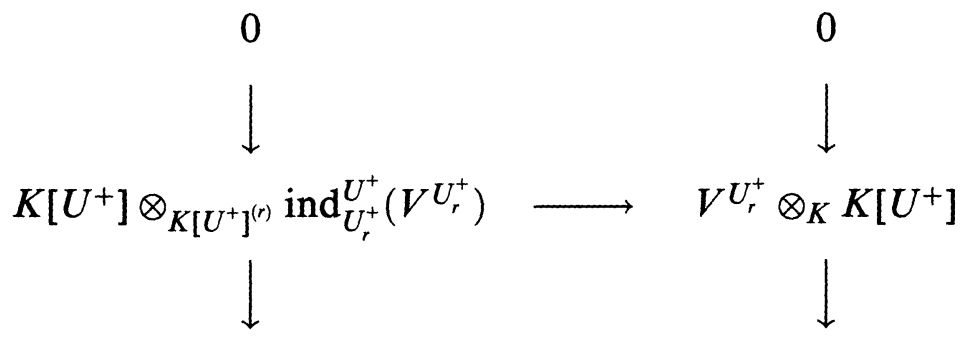

(6)

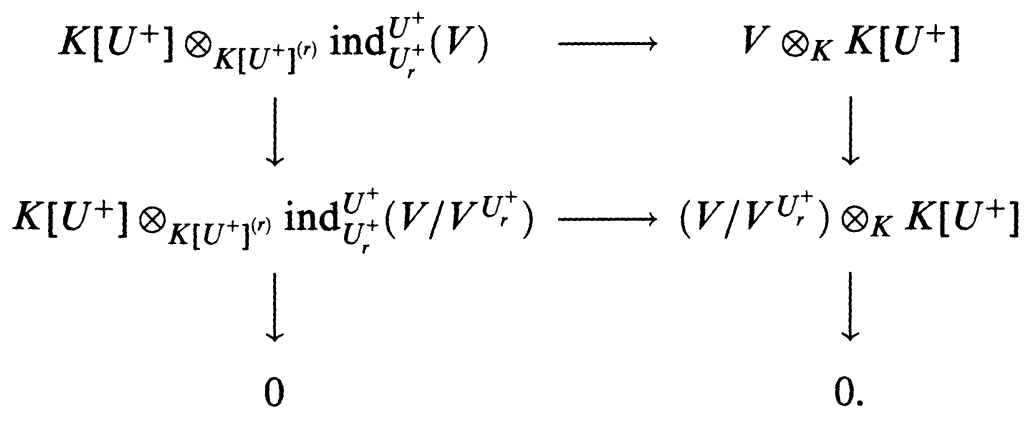

By the induction hypothesis, the top and the bottom horizontal maps are isomorphic; therefore so is the middle, as claimed.

If $s>0$, we have by the above a commutative diagram

$$
\begin{array}{cc}
\pi_{s}^{*} q_{s}^{*} \mathscr{L}_{G / G_{r+s} B}(V) \stackrel{\pi_{s}^{*} \psi}{\longrightarrow} \pi_{s}^{*} \mathscr{L}_{G / G_{s} B}(V) \\
\| & \downarrow \\
\pi_{s}^{* *} \mathscr{L}_{G / G_{r+s} B}(V) \stackrel{\sim}{\longrightarrow} & \mathscr{L}_{G / B}(V) .
\end{array}
$$

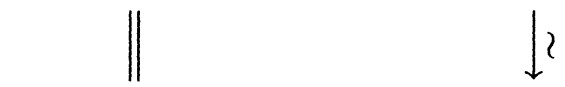

Hence at each $x \in \mathfrak{X}$ we have an isomorphism

$$
\begin{aligned}
\left(\pi_{s}^{*} \psi\right)_{x} & =\mathscr{O}_{\mathfrak{X}, x} \otimes_{\mathscr{O}_{G / G_{s} B, \pi_{s}(x)}} \psi_{\pi_{s}(x)}: \\
\mathscr{O}_{\mathfrak{X}, x} & \otimes_{\mathscr{O}_{G / G_{s} B, \pi_{s}(x)}} \mathscr{O}_{G / G_{s} B, \pi_{s}(x)} \otimes_{\mathscr{O}_{G / G_{r+s} B, \pi_{s}^{\prime}(x)}} \mathscr{L}_{G / G_{r+s} B}(V)_{\pi_{s}^{\prime}(x)} \\
& \rightarrow \mathscr{O}_{\mathfrak{X}, x} \otimes_{\mathscr{O}_{G / G_{s} B, \pi_{s}(x)}} \mathscr{L}_{G / G_{s} B}(V)_{\pi_{s}(x)} .
\end{aligned}
$$

But $\mathscr{O}_{\mathfrak{X}, x}$ is free of rank $p^{s\left|R^{+}\right|}$over $\mathscr{O}_{\mathfrak{X}^{(s)}, x}$; hence $\mathscr{O}_{\mathfrak{X}, x}$ is faithfully flat over $\mathscr{O}_{G / G_{s} B, \pi_{s}(x)}$, so $\psi_{\pi_{s}(x)}$ is already isomorphic, from which we conclude that $\psi: q_{s}^{*} \mathscr{L}_{G / G_{r+s} B}(V) \rightarrow \mathscr{L}_{G / G_{s} B}(V)$ is an isomorphism.

(2.7) One can likewise show 
Proposition. Let $(\mathfrak{G}, \mathfrak{H})=(G, B)$ or $\left(G, G_{r} B\right)$. Let $M, M^{\prime} \in$ $\mathfrak{H}$ Mod .

(i) The natural morphism

$$
\mathscr{L}_{\mathfrak{G} / \mathfrak{H}}(M) \otimes_{\mathcal{O}_{\mathfrak{B} / \mathfrak{H}}} \mathscr{L}_{\mathfrak{G} / \mathfrak{H}}\left(M^{\prime}\right) \rightarrow \mathscr{L}_{\mathfrak{G} / \mathfrak{H}}\left(M \otimes_{K} M^{\prime}\right)
$$

is invertible.

(ii) If $M$ is finite dimensional, $\mathscr{L}_{\mathfrak{G} / \mathfrak{H}}(M)$ is locally free of rank $\operatorname{dim} M$, and the natural morphism $\mathscr{L}_{\mathfrak{G} / \mathfrak{H}}\left(M^{*}\right) \rightarrow \mathscr{L}_{\mathfrak{G} / \mathfrak{H}}(M)^{\vee}$ is invertible.

(2.8) LemmA. Let $(\mathfrak{G}, \mathfrak{H})=(G, B)$ or $\left(G, G_{r} B\right)$. If $L, M$, and $N$ are $\mathfrak{H}$-modules with $L$ and $M$ finite dimensional, put

$$
\begin{aligned}
M_{1} & =\operatorname{Mod}_{\mathfrak{G} / \mathfrak{H}}\left(\mathscr{L}_{\mathfrak{G} / \mathfrak{H}}(L), \mathfrak{L}_{\mathfrak{G} / \mathfrak{H}}(M)\right), \\
M_{2} & =\operatorname{Mod}_{\mathfrak{G} / \mathfrak{H}}\left(\mathscr{L}_{\mathfrak{G} / \mathfrak{H}}(M), \mathscr{L}_{\mathfrak{G} / \mathfrak{H}}(N)\right), \\
M_{3} & =\operatorname{Mod}_{\mathfrak{G} / \mathfrak{H}}\left(\mathscr{L}_{\mathfrak{G} / \mathfrak{H}}(L), \mathscr{L}_{\mathfrak{G} / \mathfrak{H}}(N)\right) .
\end{aligned}
$$

Then one has a commutative diagram of $K$-linear spaces

$$
\begin{array}{ccc}
M_{1} \otimes_{K} M_{2} & \stackrel{c}{\longrightarrow} & M_{3} \\
2 \uparrow & \uparrow 2 & \uparrow \\
\operatorname{ind}_{\mathfrak{H}}^{\mathfrak{G}}\left(L^{*} \otimes_{K} M\right) \otimes_{K} \operatorname{ind}_{\mathfrak{H}}^{\mathfrak{G}}\left(M^{*} \otimes_{K} N\right) & \stackrel{\mu}{\longrightarrow} \operatorname{ind}_{\mathfrak{H}}^{\mathfrak{G}}\left(L^{*} \otimes_{K} N\right),
\end{array}
$$

where $c$ is the composition, the vertical isomorphisms are the natural ones, and $\mu \in \mathfrak{G}$ Mod, such that the diagram

$$
\begin{array}{ccc}
\operatorname{ind}_{\mathfrak{H}}^{\mathfrak{G}}\left(L^{*} \otimes_{K} M\right) \otimes_{K} \operatorname{ind}_{\mathfrak{H}}^{\mathfrak{G}}\left(M^{*} \otimes_{K} N\right) & \stackrel{\mu}{\longrightarrow} & \operatorname{ind}_{\mathfrak{H}}^{\mathfrak{G}}\left(L^{*} \otimes_{K} N\right) \\
\downarrow_{L^{*} \otimes_{K} M^{\otimes_{K}} e_{M^{*} \otimes_{K}}} & \\
L^{*} \otimes_{K} M \otimes_{K} M^{*} \otimes_{K} N & \stackrel{e_{L^{*} \otimes_{K^{N}}}}{\longrightarrow} & L^{*} \otimes_{K} N
\end{array}
$$

commutes if $\nu$ is the natural map.

Proof. Let $\psi_{1} \in \operatorname{ind}_{\mathfrak{H}}^{\mathfrak{G}}\left(L^{*} \otimes_{K} M\right), \psi_{2} \in \operatorname{ind}_{\mathfrak{H}}^{\mathfrak{G}}\left(M^{*} \otimes_{K} N\right), \psi_{3}=$ $\mu\left(\psi_{1} \otimes_{K} \psi_{2}\right)$, and $\tilde{\psi}_{1} \in M_{1}, \tilde{\psi}_{2} \in M_{2}, \tilde{\psi}_{3} \in M_{3}$ corresponding to $\psi_{1}, \psi_{2}, \psi_{3}$, respectively. We must show

$$
\tilde{\psi}_{2} \circ \tilde{\psi}_{1}=\tilde{\psi}_{3} \text {. }
$$

One has

$$
\psi_{3}=\left(L^{*} \otimes_{K} \nu \otimes_{K} N\right) \circ\left(\psi_{1} \otimes_{K} \psi_{2}\right) \quad \text { in } \operatorname{Sch}_{K}\left(\mathfrak{G}, L^{*} \otimes_{K} N\right) .
$$


If $\mathfrak{V}$ is an affine open of $\mathfrak{X}$, one can write $\operatorname{res}_{\pi^{-1} \mathfrak{V}}^{\mathfrak{G}}\left(\psi_{1}\right)=\sum_{i} a_{i} \otimes f_{i} \otimes m_{i} \quad$ in $K\left[\pi^{-1} \mathfrak{V}\right] \otimes_{K} L^{*} \otimes_{K} M$, and

$$
\operatorname{res}_{\pi^{-1} \mathfrak{V}}^{\mathfrak{G}}\left(\psi_{2}\right)=\sum_{j} b_{j} \otimes g_{j} \otimes n_{j} \quad \text { in } K\left[\pi^{-1} \mathfrak{V}\right] \otimes_{K} M^{*} \otimes_{K} N
$$

Then by (2)

$$
\begin{aligned}
\operatorname{res}_{\pi^{-1} \mathfrak{V}}^{\mathfrak{G}}\left(\psi_{3}\right)=\sum_{i, j} a_{i} b_{j} \otimes & g_{j}\left(m_{i}\right) f_{i} \otimes n_{j} \\
& \text { in } K\left[\pi^{-1} \mathfrak{V}\right] \otimes_{K} L^{*} \otimes_{K} N .
\end{aligned}
$$

If $v=\sum_{k} c_{k} \otimes l_{k} \in\left(K\left[\pi^{-1} \mathfrak{V}\right] \otimes_{K} L\right)^{\mathfrak{H}} \simeq \Gamma(\mathfrak{V}, \mathscr{L}(L))$, then

$$
\tilde{\psi}_{3}(\mathfrak{V})(v)=\sum_{i, j, k} a_{i} b_{j} c_{k} \otimes g_{j}\left(m_{i}\right) f_{i}\left(l_{k}\right) n_{j} \quad \text { by }(3)
$$

while

$$
\begin{aligned}
\left(\left(\tilde{\psi}_{2} \circ \tilde{\psi}_{1}\right)(\mathfrak{V})\right)(v) & =\tilde{\psi}_{2}(\mathfrak{V})\left(\sum_{i, k} a_{i} c_{k} \otimes f_{i}\left(l_{k}\right) m_{i}\right) \\
& =\sum_{i, j, k} a_{i} c_{k} b_{j} \otimes f_{i}\left(l_{k}\right) g_{j}\left(m_{i}\right) n_{j}
\end{aligned}
$$

hence $\tilde{\psi}_{2} \circ \tilde{\psi}_{1}=\tilde{\psi}_{3}$ in $\mathfrak{V}$, as desired.

(2.9) Let $M \in B$ Mod, and denote the isomorphism $\mathscr{L}_{G / G_{r} B}\left(\widehat{Z}_{r}(M)\right)$ $\rightarrow q_{*} \mathscr{L}_{G / B}(M)\left(\right.$ resp. $\left.q^{*} \mathscr{L}_{G / G_{r} B}\left(\widehat{Z}_{r}(M)\right) \rightarrow \mathscr{L}_{G / B}\left(\widehat{Z}_{r}(M)\right)\right)$ of (2.4) (resp. (2.6)) by $\theta_{1}$ (resp. $\theta_{2}$ ). One readily verifies

LEMMA. If $\tilde{a}: q^{*} q_{*} \mathscr{L}_{G / B}(M) \rightarrow \mathscr{L}_{G / B}(M)$ is the adjunction, then

$$
\tilde{a} \circ q^{*} \theta_{1}=\mathscr{L}_{G / B}\left(e_{M}\right) \circ \theta_{2} \text {. }
$$

(2.10) Let $M^{\prime}$ be another $B$-module. If $\operatorname{dim} M<\infty$, one gets from (2.9) a commutative diagram of $K$-linear spaces (1)

$$
\begin{aligned}
& \operatorname{Mod}_{\mathfrak{X}}\left(q^{*} q_{*} \mathscr{L}_{\mathfrak{X}}(M), \mathscr{L}_{\mathfrak{X}}\left(M^{\prime}\right)\right) \quad \sim \operatorname{Mod}_{\mathfrak{X}}\left(q^{*} \mathscr{L}_{G^{\prime} G_{r} B}\left(\widehat{Z}_{r}(M)\right), \mathscr{L}_{\mathfrak{X}}\left(M^{\prime}\right)\right) \\
& \operatorname{Mod}_{\mathfrak{X}}\left(\tilde{a}, \mathscr{L}_{\mathfrak{X}}\left(M^{\prime}\right)\right) \uparrow \\
& 2 \uparrow \operatorname{Mod}_{\mathfrak{X}}\left(\theta_{2}, \mathscr{L}_{\mathfrak{X}}\left(M^{\prime}\right)\right) \\
& \operatorname{Mod}_{\mathfrak{X}}\left(\mathscr{L}_{\mathfrak{X}}(M), \mathscr{L}_{\mathfrak{X}}\left(M^{\prime}\right)\right) \\
& \longrightarrow \quad \operatorname{Mod}_{\mathfrak{X}}\left(\mathscr{L}_{\mathfrak{X}}\left(\widehat{Z}_{r}(M)\right), \mathscr{L}_{\mathfrak{X}}\left(M^{\prime}\right)\right) \\
& \text { l| } \\
& \operatorname{ind}_{B}^{G}\left(M^{*} \otimes_{K} M^{\prime}\right) \\
& \underset{\operatorname{ind}_{B}^{G}\left(e_{M}^{*} \otimes_{K} M^{\prime}\right)}{\longrightarrow} \quad \operatorname{ind}_{B}^{G}\left(\widehat{Z}_{r}(M)^{*} \otimes_{K} M^{\prime}\right),
\end{aligned}
$$


where the top (resp. middle) horizontal map is $\operatorname{Mod}_{\mathfrak{X}}\left(q^{*} \theta_{1}, \mathscr{L}_{\mathfrak{X}}\left(M^{\prime}\right)\right)$ $\left(\right.$ resp. $\left.\operatorname{Mod}_{\mathfrak{X}}\left(\mathscr{L}_{\mathfrak{X}}\left(e_{M}\right), \mathscr{L}_{\mathfrak{X}}\left(M^{\prime}\right)\right)\right)$ and $e_{M}^{*} \in B \operatorname{Mod}\left(M^{*}, \widehat{Z}_{r}(M)^{*}\right)$ is the dual of $e_{M}$.

On the other hand, let $L \in G_{r} B$ Mod with $\operatorname{dim} L<\infty, \tau_{1} \in$ $G_{r} B \operatorname{Mod}\left(L^{*} \otimes_{K} \widehat{Z}_{r}\left(M^{\prime}\right), \widehat{Z}_{r}\left(L^{*} \otimes_{K} M^{\prime}\right)\right)$ the tensor identity (2.3)(7), and $\tau_{2} \in G \operatorname{Mod}\left(\operatorname{ind}_{G_{r} B}^{G}\left(\widehat{Z}_{r}\left(L^{*} \otimes_{K} \widehat{Z}_{r}\left(M^{\prime}\right)\right)\right), \operatorname{ind}_{B}^{G}\left(L^{*} \otimes_{K} M^{\prime}\right)\right)$ the transitivity of inductions $(2.4)(3)$. If $\theta_{1}^{\prime}$ (resp. $\theta_{2}^{L}$ ) is $\theta_{1}$ (resp. $\theta_{2}$ ) with $M$ (resp. $\left.\widehat{Z}_{r}(M)\right)$ replaced by $M^{\prime}$ (resp. $L$ ), one has a commutative diagram of $K$-linear spaces

$$
\begin{aligned}
& \operatorname{Mod}_{G / G_{r} B}\left(\mathscr{L}_{G / G_{r} B}(L), q_{*} \mathscr{L}_{\mathfrak{X}}\left(M^{\prime}\right)\right) \quad \sim \operatorname{Mod}_{\mathfrak{X}}\left(q^{*} \mathscr{L}_{G / G_{r} B}(L), \mathscr{L}_{\mathfrak{X}}\left(M^{\prime}\right)\right) \\
& \operatorname{Mod}_{G / G_{r} B}\left(\mathscr{L}_{G / G_{r} B}(L), \theta_{1}^{\prime}\right) \uparrow_{2} \\
& \text { (2) } \operatorname{Mod}_{G / G_{r} B}\left(\mathscr{L}_{G / G_{r} B}(L), \mathscr{L}_{G / G_{r} B}\left(\widehat{Z}_{r}\left(M^{\prime}\right)\right)\right) \\
& \text { 2 } \operatorname{Mod}_{\mathfrak{X}}\left(\theta_{2}^{L}, \mathscr{L}_{\mathfrak{X}}\left(M^{\prime}\right)\right) \\
& \operatorname{Mod}_{\mathfrak{X}}\left(\mathscr{L}_{\mathfrak{X}}(L), \mathscr{L}_{\mathfrak{X}}\left(M^{\prime}\right)\right) \\
& \text { l| } \\
& \operatorname{ind}_{G_{r} B}^{G}\left(L^{*} \otimes_{K} \widehat{Z}_{r}\left(M^{\prime}\right)\right)
\end{aligned}
$$

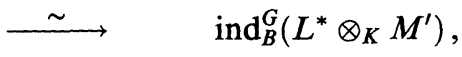

where the top (resp. bottom) isomorphism is an adjunction (resp. $\tau_{2} \circ$ $\left.\operatorname{ind}_{G_{r} B}^{G}\left(\tau_{1}\right)\right)$.

Then putting together (1) and (2) yields

(2.11) Lemma. If $M, M^{\prime} \in B$ Mod with $\operatorname{dim} M<\infty$, one has a commutative diagram of $K$-linear spaces

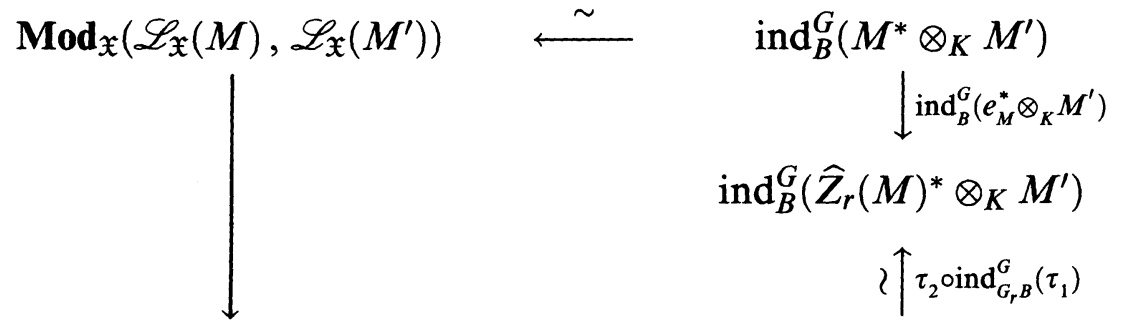

$\operatorname{Mod}_{G / G_{r} B}\left(q_{*} \mathscr{L}_{\mathfrak{X}}(M), q_{*} \mathscr{L}_{\mathfrak{X}}\left(M^{\prime}\right)\right) \stackrel{\sim}{\sim} \operatorname{ind}_{G_{r} B}^{G}\left(\widehat{Z}_{r}(M)^{*} \otimes_{K} \widehat{Z}_{r}\left(M^{\prime}\right)\right)$, where the left vertical map is given by $f \mapsto q_{*} f$,

$$
\tau_{1} \in G_{r} B \operatorname{Mod}\left(\widehat{Z}_{r}(M)^{*} \otimes_{K} \widehat{Z}_{r}\left(M^{\prime}\right), \widehat{Z}_{r}\left(\widehat{Z}_{r}(M)^{*} \otimes_{K} M^{\prime}\right)\right)
$$

is the tensor identity, and

$$
\tau_{2} \in G \operatorname{Mod}\left(\operatorname{ind}_{G_{r} B}^{G}\left(\widehat{Z}_{r}\left(\widehat{Z}_{r}(M)^{*} \otimes_{K} M^{\prime}\right)\right), \operatorname{ind}_{B}^{G}\left(\widehat{Z}_{r}(M)^{*} \otimes_{K} M^{\prime}\right)\right)
$$

is the transitivity of inductions. 


\section{Flag schemes.}

(3.1) As $F: G / G_{r} B \rightarrow \mathfrak{X}^{(r)}$ is invertible, to see that $\mathfrak{X}=G / B$ is Frobenius split, one has only to show that

$$
F_{*}^{-1}\left(\left(F_{\mathfrak{X}}^{r}\right)^{\mathfrak{f}}\right) \in \operatorname{Mod}_{G / G_{r} B}\left(\mathscr{O}_{G / G_{r} B}, q_{*} \mathscr{O}_{\mathfrak{X}}\right)
$$

admits a left inverse.

One has $\operatorname{soc}_{G_{r} B} \widehat{Z}_{r}(K)=K$ by $(2.5)(8)$; hence one has the inclusion $i \in G_{r} B \operatorname{Mod}\left(K, \widehat{Z}_{r}(K)\right)$. As $\mathscr{L}_{G / G_{r} B}$ is exact, $\mathscr{L}_{G / G_{r} B}(i)$ induces monic $\mathscr{O}_{G / G_{r} B} \rightarrow q_{*} \mathscr{O}_{\mathfrak{X}}$. On the other hand,

$$
\operatorname{Mod}_{G / G_{r} B}\left(\mathscr{O}_{G / G_{r} B}, q_{*} \mathscr{O}_{\mathfrak{X}}\right) \simeq \Gamma\left(\mathfrak{X}, \mathscr{O}_{\mathfrak{X}}\right) \simeq K .
$$

Hence we may assume

$$
F_{*}^{-1}\left(\left(F_{\mathfrak{X}}^{r}\right)^{\mathfrak{f}}\right)=\mathscr{L}_{G / G_{r} B}(i) .
$$

(3.2) TheOREM. The imbedding $\mathscr{L}_{G / G_{B}}(i)$ splits to yield

$$
q_{*} \mathscr{O}_{\mathfrak{X}} \simeq \mathscr{O}_{G / G_{r} B} \oplus \mathscr{L}_{G / G_{r} B}\left(\widehat{Z}_{r}(K) / K\right) \quad \operatorname{in} \operatorname{Mod}_{G / G_{r} B}
$$

Proof. Put $i^{\vee}=\operatorname{Mod}_{G / G_{r} B}\left(\mathscr{L}_{G / G_{r} B}(i), \mathscr{O}_{G / G_{r} B}\right)$. Our objective is to show that $i^{\vee}$ is surjective. If $i^{*} \in G_{r} B \operatorname{Mod}\left(\widehat{Z}_{r}(K)^{*}, K\right)$ is the dual of $i$, one has a commutative diagram of $K$-linear spaces

$$
\begin{array}{ccc}
\operatorname{Mod}_{G / G_{r} B}\left(\mathscr{L}_{G / G_{r} B}\left(\widehat{Z}_{r}(K)\right), \mathscr{O}_{G / G_{r} B}\right) \stackrel{i^{\vee}}{\longrightarrow} \operatorname{Mod}_{G / G_{r} B}\left(\mathscr{L}_{G / G_{r} B}(K), \mathscr{O}_{G / G_{r} B}\right) \\
\operatorname{ind}_{G_{r} B}^{G}\left(\widehat{Z}_{r}(K)^{*}\right) & \longrightarrow \\
e_{\widehat{Z}_{r}(K)^{*}} \mid & \operatorname{ind}_{G_{r} B}^{G}(K) \\
\widehat{Z}_{r}(K)^{*} & & \mid e_{K} \\
& \longrightarrow & K,
\end{array}
$$

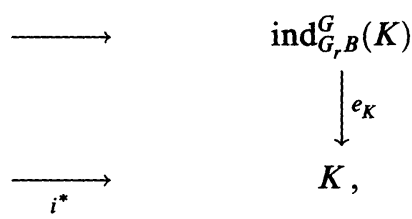

where the middle horizontal map is $\operatorname{ind}_{G_{r} B}^{G}\left(i^{*}\right)$.

As $e_{K}$ is invertible and as $i^{*}$ is surjective, one has only to show

$$
e_{\widehat{Z}_{r}(K)^{*}} \text { is surjective. }
$$

Recall the tensor identity $(2.3)(7)$

$$
S t_{r} \otimes_{K} S t_{r} \simeq \widehat{Z}_{r}\left(\left(p^{r}-1\right) \rho \otimes_{K} S t_{r}\right) \quad \text { in } G_{r} B \text { Mod. }
$$

As $\widehat{Z}_{r}$ is exact, a surjective

$$
\left(p^{r}-1\right) \rho \otimes_{K} e_{\left(p^{r}-1\right) \rho} \in B \operatorname{Mod}\left(\left(p^{r}-1\right) \rho \otimes_{K} S t_{r}, 2\left(p^{r}-1\right) \rho\right)
$$


induces a surjective

$$
\begin{aligned}
& \widehat{Z}_{r}\left(\left(p^{r}-1\right) \rho \otimes_{K} e_{\left(p^{r}-1\right) \rho}\right) \\
& \quad \in G_{r} B \operatorname{Mod}\left(S t_{r} \otimes_{K} S t_{r}, \widehat{Z}_{r}\left(2\left(p^{r}-1\right) \rho\right)\right) .
\end{aligned}
$$

But $\widehat{Z}_{r}\left(2\left(p^{r}-1\right) \rho\right) \simeq \widehat{Z}_{r}(K)^{*}$ by $(2.5)(7)$; hence one gets a surjective

$$
\phi \in G_{r} B \operatorname{Mod}\left(S t_{r} \otimes_{K} S t_{r}, \widehat{Z}_{r}(K)^{*}\right) .
$$

Then $\phi$ induces $\hat{\phi} \in G \operatorname{Mod}\left(S t_{r} \otimes_{K} S t_{r}, \operatorname{ind}_{G_{r} B}^{G}\left(\widehat{Z}_{r}(K)^{*}\right)\right)$ by the Frobenius reciprocity such that

$$
e_{\widehat{Z}_{r}(K)^{*}} \circ \hat{\phi}=\phi
$$

hence $e_{\widehat{Z}_{r}(K)^{*}}$ must be surjective, as desired.

(3.3) REMARKs. (i) Unlike the case of the projective spaces, $q_{*} \mathscr{\sigma}_{G / B}$ does not in general split into a direct sum of invertible sheaves [Haas], (4.5.5).

(ii) If $s \in \mathbf{N}$, one can make as in (2.5)(11) a $G_{r} B$-module $M$ into a $G_{r+s} B$-module, denoted also by $M^{[s]}$. Then in $G_{r+s} B$ Mod

$$
\operatorname{ind}_{G_{s} B}^{G_{r+s} B}(K) \simeq \operatorname{ind}_{G_{s} B / G_{s}}^{G_{r+s} B / G_{s}}(K) \simeq\left(\operatorname{ind}_{B}^{G_{r} B}(K)\right)^{[s]} .
$$

If $q_{s}: G / G_{s} B \rightarrow G / G_{r+s} B$ is the natural morphism, one has commutative diagram in $\mathbf{S c h}_{K}$

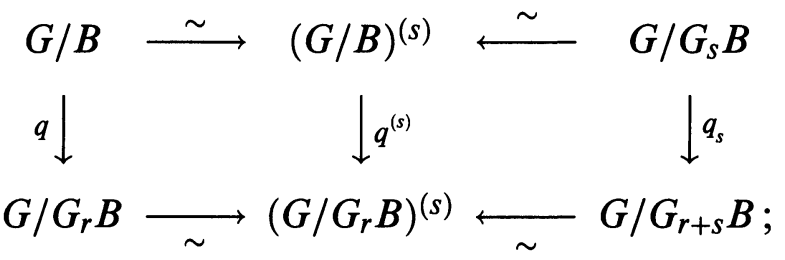

hence the natural morphism $\mathscr{O}_{G / G_{r+s} B} \rightarrow q_{s *} \mathscr{Q}_{G / G_{s} B}$ splits to yield

$$
q_{s *} \mathscr{O}_{G / G_{s} B} \simeq \mathscr{O}_{G / G_{r+s} B} \oplus \mathscr{L}_{G / G_{r+s} B}\left(\left(\widehat{Z}_{r}(K) / K\right)^{[s]}\right)
$$

in $\operatorname{Mod}_{G / G_{r+s} B}$.

(iii) The cup product $S t_{r} \otimes_{K} S t_{r} \rightarrow \operatorname{ind}_{B}^{G}\left(2\left(p^{r}-1\right) \rho\right)$ in $G$ Mod, induced by the multiplication $\left(p^{r}-1\right) \rho \otimes_{K}\left(p^{r}-1\right) \rho \rightarrow 2\left(p^{r}-1\right) \rho$, turns out to be surjective (cf. [J], (II.14.20)). On the other hand, one 
has $K$-linear isomorphisms

$$
\begin{gathered}
G \operatorname{Mod}\left(S t_{r} \otimes_{K} S t_{r}, \operatorname{ind}_{G_{r} B}^{G}\left(\widehat{Z}_{r}(K)^{*}\right)\right) \\
\simeq G \operatorname{Mod}\left(S t_{r} \otimes_{K} S t_{r}, \operatorname{ind}_{G_{r} B}^{G}\left(\widehat{Z}_{r}\left(2\left(p^{r}-1\right) \rho\right)\right)\right) \\
\simeq G \operatorname{Mod}\left(S t_{r} \otimes_{K} S t_{r}, \operatorname{ind}_{B}^{G}\left(2\left(p^{r}-1\right) \rho\right)\right) \\
\quad \text { by the transitivity of inductions } \\
\simeq B \operatorname{Mod}\left(S t_{r} \otimes_{K} S t_{r}, 2\left(p^{r}-1\right) \rho\right) \\
\simeq K . \\
\text { by the Frobenius reciprocity }
\end{gathered}
$$

It follows that $\hat{\phi}$ is surjective, hence every morphism $q_{*} \mathscr{O}_{\mathfrak{X}} \rightarrow \mathscr{O}_{G / G_{r} B}$, and, a fortiori, every Frobenius splitting of $G / B$, is provided by $S t_{r} \otimes_{K} S t_{r}$.

\section{Schubert schemes.}

(4.1) Let $\tilde{\phi}$ be the $K$-linear map

$$
S t_{r} \otimes_{K} S t_{r} \rightarrow \operatorname{Mod}_{G / G_{r} B}\left(q_{*} \mathscr{O}_{\mathfrak{X}}, \mathscr{O}_{G / G_{r} B}\right)
$$

induced by $\hat{\phi}$ of (3.2). One has $K$-linear isomorphisms

(1) $G_{r} B \operatorname{Mod}\left(S t_{r} \otimes_{K} S t_{r}, K\right)$

$$
\begin{aligned}
& \simeq G_{r} B \operatorname{Mod}\left(S t_{r}, S t_{r}\right) \text { as } S t_{r} \text { is self-dual } \\
& \simeq B \operatorname{Mod}\left(S t_{r},\left(p^{r}-1\right) \rho\right) \text { by the Frobenius reciprocity } \\
& \simeq K \text { as }\left(p^{r}-1\right) \text { is the highest weight of } S t_{r} \\
& \simeq G \operatorname{Mod}\left(S t_{r}, S t_{r}\right) \simeq G \operatorname{Mod}\left(S t_{r} \otimes_{K} S t_{r}^{*}, K\right)=K \operatorname{Tr},
\end{aligned}
$$

where $\operatorname{Tr}$ is the trace map of the $K$-linear endomorphisms of $S t_{r}$. Hence we may assume in (3.2)

$$
i^{*} \circ \phi=\operatorname{Tr} .
$$

In particular, if $v_{-} \in S t_{r,-\left(p^{r}-1\right) \rho} \backslash 0$ and $v_{+} \in S t_{r,\left(p^{r}-1\right) \rho} \backslash 0$, then

$$
\operatorname{Tr}\left(v_{-} \otimes v_{+}\right) \neq 0
$$

as one can regard $v_{+}$as the dual of $v_{-}$. Hence one can take the splitting of $\mathscr{L}_{G / G_{r} B}(i)$ to be

$$
\sigma=\tilde{\phi}\left(v_{-} \otimes v_{+}\right)
$$

We will show 
(4.2) Theorem. Let $w \in W$. If $\mathscr{I}_{w}$ is the ideal sheaf of the Schubert scheme $\mathfrak{X}(w)=\overline{U^{+} w B / B}$ in $\mathfrak{X}$, then

$$
\sigma\left(q_{*} \mathscr{J}_{w}\right) \subseteq F_{*}^{-1}\left(\mathscr{J}_{w}^{(r)}\right) .
$$

Hence $F_{*} \sigma$ splits all the Schubert subschemes of $\mathfrak{X}$.

(4.3) Let $\mathfrak{Y}$ be a closed subscheme of $\mathfrak{X}$ with the underlying topological space $|\mathfrak{X}||| U^{+} B / B \mid$. If $\alpha$ is a simple root, the Schubert scheme $\mathfrak{X}\left(s_{\alpha}\right)$ is an irreducible component of $\mathfrak{Y}$. In $w \in W$ with $l(w) \geq 2$, there are simple roots $\alpha_{1}$ and $\alpha_{2}$ such that $l\left(s_{\alpha_{1}} w s_{\alpha_{2}}\right)=l(w)-2$. Then

$$
s_{\alpha_{1}} w \neq w s_{\alpha_{2}} \quad \text { with } l\left(s_{\alpha_{1}} w\right)=l\left(w s_{\alpha_{2}}\right)=l(w)-1 .
$$

It follows that

(2) $\mathfrak{X}(w)$ is an irreducible component of $\mathfrak{X}\left(s_{\alpha_{1}} w\right) \cap \mathfrak{X}\left(w s_{\alpha_{2}}\right)$.

Hence in order to get (4.2), it will suffice by (2.2) to show that

$$
F_{*} \sigma \text { splits } \mathfrak{Y} \text {. }
$$

(4.4) Let $j \in \operatorname{ind}_{B}^{G}(\rho)_{\rho}$ be such that $j=1$ in $U^{+}$regarded as an element of $\operatorname{Sch}_{K}(G, \rho)_{\rho}^{B}[\mathrm{~J}],($ II.2.6), and let

$$
\tilde{j} \in \operatorname{Mod}_{\mathfrak{X}}\left(\mathscr{L}_{\mathfrak{X}}(-\rho), \mathscr{O}_{\mathfrak{X}}\right) \simeq \Gamma\left(\mathfrak{X}, \mathscr{L}_{\mathfrak{X}}(\rho)\right)
$$

corresponding to $j$. If $w \in W$, one has a commutative diagram

$$
\Gamma\left(w U^{+} B / B, \mathscr{L}_{\mathfrak{X}}(-\rho)\right) \stackrel{\tilde{j}\left(w U^{+} B / B\right)}{\longrightarrow} \Gamma\left(w U^{+} B / B, \mathscr{O}_{\mathfrak{X}}\right)
$$

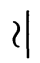

(1)

$$
\begin{aligned}
& \operatorname{Sch}_{K}\left(w U^{+},-\rho\right) \\
& \text { 2) } \\
& K\left[w U^{+}\right] \\
& \underset{\left.j\right|_{w U^{+}}}{\longrightarrow} \quad K\left[w U^{+}\right] .
\end{aligned}
$$

If $\left.j\right|_{w U^{+}}=0$, then $j$ would vanish in $w U^{+} B$ that is open in $G$, hence in the whole of $G$, contradicting the choice of $j$. It follows that

$$
\tilde{j} \text { is monic. }
$$



(4.5)
LemMA. Supp $(\operatorname{coker} \tilde{j})=|\mathfrak{X}| \backslash\left|U^{+} B / B\right|$.

Proof. As $\tilde{j}$ is invertible in $U^{+} B / B$,

$$
\operatorname{Supp}(\operatorname{coker} \tilde{j}) \subseteq|\mathfrak{X}| \backslash\left|U^{+} B / B\right| .
$$

On the other hand, if $\alpha$ is a simple root, one finds $j=0$ in $U^{+} s_{\alpha} B$ (cf. [J], (II.2.6)); hence

$$
\operatorname{Supp}(\operatorname{coker} \tilde{j}) \supseteq\left|U^{+} s_{\alpha} B / B\right| .
$$

But $\operatorname{Supp}(\operatorname{coker} \tilde{j})$ is closed in $\mathfrak{X}$ as $\mathscr{L}_{\mathfrak{X}}(-\rho)$ is quasicoherent. Hence

$$
\begin{aligned}
\operatorname{Supp}(\operatorname{coker} \tilde{j}) & \supseteq \bigcup_{\alpha \text { simple }}\left|\mathfrak{X}\left(s_{\alpha}\right)\right| \\
& =\bigcup_{w \in W \backslash 1}\left|U^{+} w B / B\right|=|\mathfrak{X}| \backslash\left|U^{+} B / B\right|,
\end{aligned}
$$

and the assertion follows.

(4.6) We take $\mathfrak{Y}$ to be the closed subscheme of $\mathfrak{X}$ defined by the ideal sheaf $\operatorname{im} \tilde{j}$. One has a commutative diagram of short exact sequences
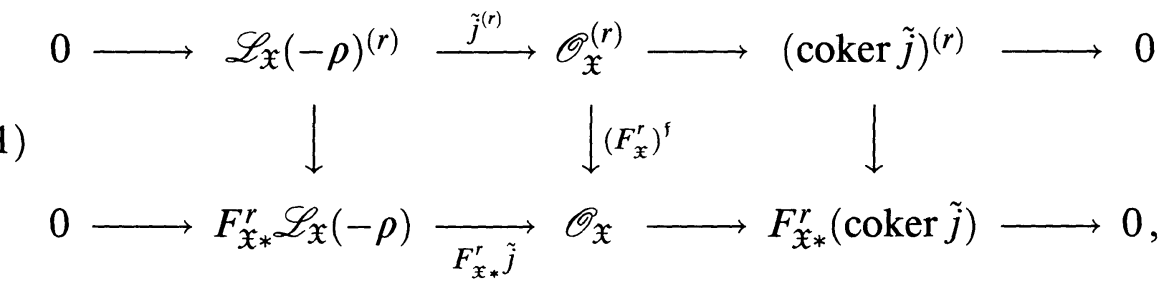

where the left vertical morphism is given by $f \mapsto f^{p^{r}} j^{p^{r}-1}$. If $\tilde{j}_{r}=$ $F_{*}^{-1}\left(\tilde{j}^{(r)}\right)$, hitting $F_{*}^{-1}$ on (1) yields by $(2.5)(11)$ a commutative diagram of short exact sequences

(2)

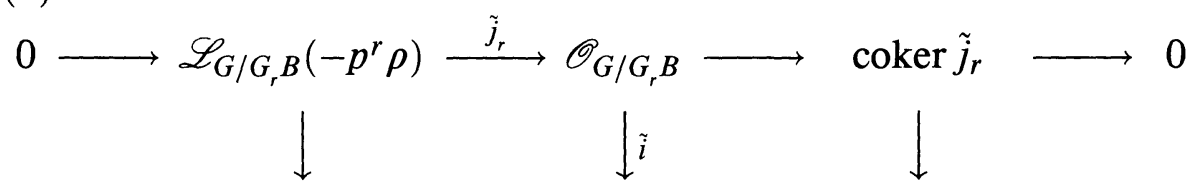

$0 \longrightarrow q_{*} \mathscr{L}_{\mathfrak{X}}(-\rho) \quad \underset{q_{*} \tilde{j}}{\longrightarrow} q_{*} \mathscr{O}_{\mathfrak{X}} \longrightarrow q_{*}(\operatorname{coker} \tilde{j}) \longrightarrow 0$,

with $j_{r}=\tilde{j}_{r}\left(G / G_{r} B\right) \in \operatorname{ind}_{G_{r} B}^{G}\left(p^{r} \rho\right)_{p^{r}} \rho \backslash 0$.

Our objective is to show 
(4.7) Proposition. $\sigma\left(\operatorname{im}\left(q_{*} \tilde{j}\right)\right) \subseteq \operatorname{im} \tilde{j}_{r}$.

Proof. Put

(1)

$$
\begin{aligned}
M_{1} & =\operatorname{Mod}_{G / G_{r} B}\left(q_{*} \mathscr{O}_{\mathfrak{X}}, \mathscr{O}_{G / G_{r} B}\right), \\
M_{2} & =\operatorname{Mod}_{G / G_{r} B}\left(q_{*} \mathscr{L}_{\mathfrak{X}}(-\rho), q_{*} \mathscr{O}_{\mathfrak{X}}\right), \\
M_{3} & =\operatorname{Mod}_{G / G_{r} B}\left(q_{*} \mathscr{L}_{\mathfrak{X}}(-\rho), \mathscr{L}_{G / G_{r} B}\left(-p^{r} \rho\right)\right), \\
M_{4} & =\operatorname{Mod}_{G / G_{r} B}\left(\mathscr{L}_{G / G_{r} B}\left(-p^{r} \rho\right), \mathscr{O}_{G / G_{r} B}\right), \\
I_{1} & =\operatorname{ind}_{G_{r} B}^{G}\left(\widehat{Z}_{r}(K)^{*}\right), \\
I_{2} & =\operatorname{ind}_{G_{r} B}^{G}\left(\widehat{Z}_{r}(K) \otimes_{K} \widehat{Z}_{r}(-\rho)^{*}\right) .
\end{aligned}
$$

One has in $G$ Mod

(2) $\quad \operatorname{ind}_{G_{r} B}^{G}\left(\widehat{Z}_{r}(-\rho)^{*}\right) \simeq \operatorname{ind}_{G_{r} B}^{G}\left(\widehat{Z}_{r}\left(\left(2 p^{r}-1\right) \rho\right)\right)$ by $(2.5)(7)$

$$
\simeq \operatorname{ind}_{B}^{G}\left(\left(2 p^{r}-1\right) \rho\right) \text { by the transitivity of inductions }
$$

and

$$
\begin{aligned}
\operatorname{ind}_{G_{r} B}^{G} & \left(\widehat{Z}_{r}(-\rho)^{*} \otimes_{K}-p^{r} \rho\right) \\
& \simeq \operatorname{ind}_{G_{r} B}^{G}\left(\widehat{Z}_{r}\left(\left(2 p^{r}-1\right) \rho\right) \otimes_{K}-p^{r} \rho\right) \\
& \simeq \operatorname{ind}_{G_{r} B}^{G}\left(\widehat{Z}_{r}\left(\left(p^{r}-1\right) \rho\right)\right) \text { by the tensor identity } \\
& \simeq S t_{r} \quad \text { by the tensor identity again. }
\end{aligned}
$$

Hence one gets by (2.8) and (2.11) a commutative diagram of $K$-linear spaces

(4)

$$
\begin{array}{rlrl}
S t_{r} \otimes_{K} S t_{r} \otimes_{K} \operatorname{Mod}_{\mathfrak{X}}\left(\mathscr{L}_{\mathfrak{X}}(-\rho), \mathscr{O}_{\mathfrak{X}}\right) & \stackrel{\sim}{ } S t_{r} \otimes_{K} S t_{r} \otimes_{K} \operatorname{ind}_{B}^{G}(\rho) \\
\tilde{\phi} \otimes_{K} q_{*} \downarrow & \stackrel{\sim}{ } & \downarrow_{\hat{\phi} \otimes_{K} \nu_{1}} \\
M_{1} \otimes_{K} M_{2} & & I_{1} \otimes_{K} I_{2} \\
c_{1} \downarrow & \stackrel{\nu_{2}}{\sim} & \operatorname{ind}_{B}^{G}\left(\left(2 p^{r}-1\right) \rho\right) \\
\operatorname{Mod}_{G / G_{r} B}\left(q_{*} \mathscr{L}_{\mathfrak{X}}(-\rho), \mathscr{O}_{G / G_{r} B}\right) & \longleftarrow & \uparrow_{\nu_{3}} \\
c_{2} \uparrow & & \sim & \operatorname{St}_{r} \otimes_{K} \operatorname{ind}_{G_{r} B}^{G}\left(p^{r} \rho\right),
\end{array}
$$

where $c_{1}$ and $c_{2}$ are compositions, and $\nu_{1}, \nu_{2}, \nu_{3}$ are some nonzero $G$-homomorphisms. 
If $\tilde{v}_{-} \in M_{3}$ is $v_{-}$under the isomorphism (3), then (4.7) will follow from

$$
c_{1} \circ\left(\tilde{\phi} \otimes_{K} q_{*}\right)\left(v_{-} \otimes_{K} v_{+} \otimes_{K} \tilde{j}\right)=c_{2}\left(\tilde{v}_{-} \otimes_{K} \tilde{j}_{r}\right),
$$

which translates through (4) into

$$
\nu_{2} \circ\left(\hat{\phi} \otimes_{K} \nu_{1}\right)\left(v_{-} \otimes_{K} v_{+} \otimes_{K} j\right)=\nu_{3}\left(v_{-} \otimes_{K} j_{r}\right) .
$$

We actually need (6) to hold only up to $K^{\times}$.

Consider an imbedding $\hat{e}_{p^{r} \rho} \in G \operatorname{Mod}\left(\operatorname{ind}_{G_{r} B}^{G}\left(p^{r} \rho\right), \operatorname{ind}_{B}^{G}\left(p^{r} \rho\right)\right)$ and put $j_{r}^{\prime}=\hat{e}_{p^{r} \rho}\left(j_{r}\right)$. One has $K$-linear isomorphisms

$$
\begin{aligned}
G & \operatorname{Mod}\left(S t_{r} \otimes_{K} S t_{r} \otimes_{K} \operatorname{ind}_{B}^{G}(\rho), \operatorname{ind}_{B}^{G}\left(\left(2 p^{r}-1\right) \rho\right)\right) \\
& \simeq B \operatorname{Mod}\left(S t_{r} \otimes_{K} S t_{r} \otimes_{K} \operatorname{ind}_{B}^{G}(\rho),\left(2 p^{r}-1\right) \rho\right) \\
& \simeq K \quad \text { by the Frobenius reciprocity } \\
& \simeq G \operatorname{Mod}\left(S t_{r} \otimes_{K} \operatorname{ind}_{G_{r} B}^{G}\left(p^{r} \rho\right), \operatorname{ind}_{B}^{G}\left(\left(2 p^{r}-1\right) \rho\right)\right) \\
& \simeq G \operatorname{Mod}\left(S t_{r} \otimes_{K} \operatorname{ind}_{B}^{G}\left(p^{r} \rho\right), \operatorname{ind}_{B}^{G}\left(\left(2 p^{r}-1\right) \rho\right)\right) .
\end{aligned}
$$

Hence if $\mu_{1} \in G \operatorname{Mod}\left(S t_{r} \otimes_{K} S t_{r} \otimes_{K} \operatorname{ind}_{B}^{G}(\rho), \operatorname{ind}_{B}^{G}\left(\left(2 p^{r}-1\right) \rho\right)\right)$ and $\mu_{2} \in G \operatorname{Mod}\left(S t_{r} \otimes_{K} \operatorname{ind}_{B}^{G}\left(p^{r} \rho\right), \operatorname{ind}_{B}^{G}\left(\left(2 p^{r}-1\right) \rho\right)\right)$ are the cup products, we are reduced to showing

$$
\mu_{1}\left(v_{-} \otimes_{K} v_{+} \otimes_{K} j\right)=\mu_{2}\left(v_{-} \otimes_{K} j_{r}^{\prime}\right) \quad \text { up to } K^{\times} .
$$

But we have another cup product

$$
\mu_{3} \in G \operatorname{Mod}\left(S t_{r} \otimes_{K} \operatorname{ind}_{B}^{G}(\rho), \operatorname{ind}_{B}^{G}\left(p^{r} \rho\right)\right)
$$

such that

$$
\mu_{1}=\mu_{2} \circ\left(S t_{r} \otimes_{K} \mu_{3}\right) .
$$

By the weight consideration we must have

$$
\mu_{3}\left(v_{+} \otimes j\right)=j_{r}^{\prime} \quad \text { up to } K^{\times} ;
$$

hence (8) follows, as desired.

(4.8) Finally, if $P$ is a parabolic subgroup of $G$ containing $B$, let $\bar{\pi}: G / B \rightarrow G / P$ be the natural morphism. As $G / B$ and $G / P$ are both projective over $K, \bar{\pi}$ is projective. Also $\bar{\pi}_{*} \mathscr{O}_{G / B}=\mathscr{O}_{G / P}$, as $\bar{\pi}$ is locally trivial with $\mathscr{O}_{P / B}(P / B)=K$. Hence one gets from [MR], Proposition 4, 
CoRollary. $\bar{\pi}_{*}\left(F_{*} \sigma\right)$ splits all the Schubert subshcemes of $G / P$.

\section{REFERENCES}

[CPS] E. Cline, B. Parshall and L. Scott, A Mackey imprimitivity theory for algebraic groups, Math. Z., 182 (1983), 447-471.

[Haas] B. Haastert, Über Differentialoperatoren und $\mathscr{D}$-moduln in positiver Charakteristik, Manuscripta Math., 58 (1987), 385-415.

[HASV] R. Hartshorne, Ample Subvarieties of Algebraic Varieties, Springer LNM, 156 (1970).

[J] J. C. Jantzen, Representations of Algebraic Groups, Academic Press, Orlando, 1987.

[MR] V. B. Mehta and A. Ramanathan, Frobenius splitting and cohomology vanishing for Schubert varieties, Ann. Math., 122 (1985), 27-40.

[R] A. Ramanathan, Equations defining Schubert varieties and Frobenius splitting of diagonals, IHES Pub. Math., 65 (1987), 61-90.

Received January 27, 1992 and in revised form April 19, 1993.

DePartment of MATHEMATics

FACULTY OF SCIENCE

NigATA UNIVERSITY

NigAta 950-21 JAPAN

E-mail address: F00500@sinet.ad.jp 


\section{CONTENTS}

D. Bisch, A note on intermediate subfactors , . , . . . . . . . . . . . . . . . . 201

M. J. Carro and J. Soria, Tent spaces over general approach regions and pointwise

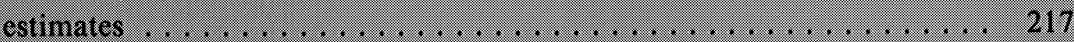

J. A. Charris, M. E. H. Ismail, and S. Monsalve, On sieved orthogonal polynomials X: general blocks of recurrence relations . . . . . . . . . . . . . . . . 237

K. S. Chou and T. Y. H. Wan, Asymptotic radial symmetry for solutions of $\Delta u+$ $e^{\prime \prime}=0$ in a punctured disc . . . . . . . . . . . . . . . . . . . . . . . 269

M. E. Fogel, Knots with algebraic unknotting number one . . . . . . . . . . . . . 277

K. N. Jones, The structure of closed nonpositively curved Euclidean cone 3-manifolds . . . . . . . . . . . . . . . . . . . . . . . . . . . . . . . . 297

M. Kaneda, On the Frobenius morphism of flag schemes . . . . . . . . . . 315

H. T. Kaptanoğlu, Möbius-invariant Hilbert spaces in polydiscs . . . . . . . . . 337

K. Kuribayashi, The cohomology ring of the spaces of loops on Lie groups and homogeneous spaces ............................ 361

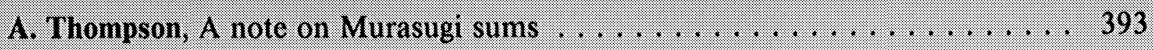




\title{
PACIFIC JOURNAL OF MATHEMATICS
}

\author{
Volume $163 \quad$ No. $2 \quad$ April 1994
}

A note on intermediate subfactors

201

DIETMAR BISCH

Tent spaces over general approach regions and pointwise estimates

217

MARÍA J. CARRO and JAVIER SORIA

On sieved orthogonal polynomials. X. General blocks of recurrence

237 relations

JAIro A. Charris, Mourad ISMAIL and SERGio Monsalve

Asymptotic radial symmetry for solutions of $\Delta u+e^{u}=0$ in a punctured disc

Kai Seng (Kaising) Chou (Tso) and Tom YaU-Heng Wan

Knots with algebraic unknotting number one

MicAh Elton Fogel

The structure of closed non-positively curved Euclidean cone

3-manifolds

KERRY NELSON JONES

On the Frobenius morphism of flag schemes

MASAHARU KANEDA

Möbius-invariant Hilbert spaces in polydiscs

H. TuRgay KaptanOGLU

The cohomology ring of the spaces of loops on Lie groups and homogeneous spaces

KATSUHIKO KURIBAYASHI

A note on Murasugi sums

ABIGAIL A. THOMPSON 